\title{
IMPROVING THE WORLD'S HEALTH - THE ROLE OF NATIONAL PUBLIC HEALTH INSTITUTES
}

\author{
Pekka Jousilahti \\ National Public Health Institute, Finland \\ Secretary General, International Association of National Public Health Institutes
}

Address for correspondence: P. Jousilahti, National Public Health Institute-KTL, Mannerheimintie 166, FIN-00300 Helsinki, Finland, E-mail: pekka.jousilahti@ktl.fi

Considerable technical capacity and experience is required to deal with global health threats, including infectious diseases such as SARS and avian influenza, and behavioural risk factors for non-communicable diseases, such as tobacco use, physical inactivity and unhealthy diet, and their health consequences including obesity, diabetes and cardiovascular disease. Other big challenges are injury prevention and preparedness and management of man made and natural disasters. Many countries have found the critical mass of skills and knowledge that can be developed in a National Public Health Institute (NPHI) to be crucially important in dealing with these health problems in a population and community.

First NPHIs, or their precursors, were established over 100 years ago. As an example, the Brazil's FIOCRUZ was established in 1900, the Finland's KTL is over 90 years old, and the Czech Institute of Public Health, which recently had its 80th anniversary, is also one of the oldest ones. Because the definitions and names of institutes vary between countries, we don't know the exact number of NPHIs in the world. About 40 institutes have been participating in the recently started international collaboration, and we can estimate that maybe half of the countries have or is currently developing a NPHI. In the early stages NPHIs were mainly dealing with the control of infectious disease. However, due to epidemiological transition and changing disease patterns, many institutes have broadened their scope to cover also non-communicable diseases and environmental health issues.

Globalisation is as applicable to health issues as to those of trade and economics.

The increased frequency of travel, distribution of goods, migration, spread of communications and marketing of new lifestyles have promoted a set of risks and health challenges shared by all countries of the world, despite their varied resources, levels of development, demographics, and other important considerations. Because microbes don't recognise borders, countries have a long tradition in international and bilateral collaboration in communicable disease control.
However, not only microbes, but also other health threats cross the borders nowadays. Two major causes on non-communicable diseases, tobacco smoking and unhealthy diet are good examples of global health threatening issues. The ability of one country to solve these new challenges on its own is increasingly difficult and certainly inefficient.

Although varying in size, historical background and administrative structure, NPHIs have many common elements. The core elements of a NPHI are given in the Table 1. Even though not all of the NPHIs cover all of these aspects, the role and function of a NPHI is usually wider compared to universities, schools of public health, and departments of ministries. These fundamental core capacities will be important contributors to ensure public health in the 21st century.

Research is needed to create a firm knowledge base in order to anticipate future trends, needs and challenges, taking into account direct indicators of health and also structural, behavioural and social health determinants. The primary goal of research carried out in a NPHI is promotion of health and reduction of the risks of diseases of public health importance. The research function can also involve the assimilation of knowledge generated by other investigators and basic research in collaboration with universities and other partners.

Monitoring of health status and health determinants of population groups is a crucial function of the NPHIs. Data on the occurrence of non-communicable and communicable diseases and their determinants, such as smoking prevalence, blood pressure and serum cholesterol levels and immunization coverage, are needed for effective diseases prevention and health care planning. Collection of data and monitoring of disease trends and determinants, however, are not enough. Health information must be easily accessible to policy-makers, health professionals, the media and general public, all of whom are in need to make informed decisions on policies and personal health choices. 
Table 1. Core elements* of a National Public Health Institute
National
- Country wide
- May have also regional units
- International collaboration

\section{Multidisciplinary \\ - Medicine \\ - Public Health \\ - Nutrition \\ - Laboratory sciences \\ - Social sciences \\ - Health economics \\ - Statistics}

\section{Comprehensive}

- Infectious diseases

- Chronic non-communicable diseases

- Environmental health

- Occupational health

- Disaster preparedness

\author{
Multifunctional \\ - Research \\ - Monitoring and surveillance \\ - Training \\ - Public health services \\ - Diagnostic and other health services \\ - Health promotion and communication \\ - Vaccine and drug production and safety control
}

*The list is neither comprehensive nor all of the Institutes have all of these elements

Infectious disease control is a classical task of NPHIs. In every country the burden and threat posed by infectious diseases calls major public attention to prevention and control of epidemics. The development and implementation of surveillance programmes, epidemiological investigations, standardised laboratory practices, technical expertise, and support to local and regional health authorities are all important responsibilities of the NPHI. Many institutes are also involved in national immunization programmes and vaccine production.

Global health issues and the field of public health are in rapid transition. According to the World Health Report, $60 \%$ of all deaths in the world are attributable to chronic non-communicable diseases. Therefore, preventing chronic diseases is a vital investment for every society. Half of these deaths are due to cardiovascular diseases, and the other major non-communicable diseases include diabetes, cancers and chronic respiratory diseases. The increase in non-communicable disease burden is a result of the rapid expansion of risk factors and changes in lifestyles, such as diet, physical activity and smoking habit. In the western countries about $40 \%$ of people are overweight and $20 \%$ obese. Smoking prevalence vary between $25 \%$ and $60 \%$, and despite the decreasing trend among men, tobacco smoking is increasing among women in many countries. The role of NPHIs in the prevention of chronic non-communicable diseases include disease and risk factor surveillance, and also research and various public health functions, such as health promotion and education.

Major epidemics, terrorism, environmental health disasters, natural disasters, and other public health emergencies require a public health system that is responsive and capable of delivering emergency services to the affected population. Core functions include comprehensive emergency response plans, epidemiological expertise in disease investigation, and effective communication systems for information dissemination to public and health care providers. Intersectoral collaboration and cross-training is a requirement for effective emergency preparedness.

Many NPHIs are also providers of diagnostic services. Core capacities for public health laboratories depend upon the needs and functions of each country's health care delivery structure. Many NPHI laboratories coordinate national screening programmes, particularly in maternal and child health care, and serve as a reference or have a consultative role in relation to other private and public laboratories. Environmental health related laboratory analyses are also often the task of NPHIs. In some countries, NPHIs and their regional subsidiaries provide clinical laboratory services for health care services.

Most NPHIs participate in some way in disseminating health information to the public. Public attitudes towards health promotion and disease prevention are critical for the success of health campaigns and the implementation of health policy. The media also plays a key role in delivering health messages and influencing health policy. Health communication functions include expertise to provide health messages to diverse populations, implementation of evidence-based health programmes, targeted public health surveillance and assessment, and building of key coalitions and partnerships with both private and public sector to promote health and influence public opinion. New communication technologies, particularly internet, have an increasing role in the future health information dissemination. 
Training to create a competent workforce in public health takes place with the overall education and training system of the country and the role of NPHIs in the training activities vary. Usually NPHIs do the training in collaboration with universities and schools of medicine and public health. NPHIs are involved in graduate, postgraduate and in-service training of different categories of health professionals. In many countries the experts of the NPHIs are known to have a strong practical and theoretical knowledge on different public health issues, and even without a formal linkage they are often used as lecturers in educational institutions.

Although the NPHIs vary in their roles and functions, the value, service and credibility they add to the ministry of health and their country are significant. While a NPHI of the 1950s would have consisted primarily of infectious disease epidemiologists, laboratory staff and administrative staff, the present day NPHI has a mix of public health professionals with skills in laboratory science, research, health promotion, nutrition, environmental health, behavioural and social sciences, economics and communication technology, and often in many other fields.

It is important for the ministries of health around the world to be able to respond to health threatening situations rapidly and effectively, using the best public health interventions available. From the many different models of NPHI, they all have the capacity for research, public health expertise, health monitoring and service delivery, even though the breadth and depth of these functions may vary. Furthermore, a NPHI is primarily a professional and scientific organization without direct political affinity, which guarantees the continuity of the work at times of political changes.
In general, advantages of the NPHIs include an assembly of a stable mass of expertise, continuity of work, firm scientific knowledge and appropriate human, technical, and financial resources to tackle public health challenges in each country. Also in those countries, which do not have a NPHI at the moment, there is an increasing tendency to merge different entities and develop more comprehensive institutes to cover the different aspects of public health.

Close international collaboration is essential for public health protection and tackling the future health threats in the world. The directors of selected NPHIs met first time in Italy in 2002, and the group reconvened in 2004 in Finland and declared its intention to establish an International Association of National Public Health Institutes (IANPHI). The Association will be formally launched in Brazil at the beginning of 2006. The domain of the new association will include mechanisms for swift and clear communication between members, joint training activities, collaborative research projects, and mutual assistance on wide variety of public health issues such as surveillance and outbreak investigation. In an increasingly globalized world, international collaboration between NPHIs is vital for success in any country and for global public health.

This article has been presented in the 80th Anniversary Seminar of the Czech Institute of Public Health in November 2005 and it is partly based on a previous publication: Koplan JP, Puska P, Jousilahti P, Cahill $K$, Huttunen $J$ and the National Public Health Institute partners: Improving the world's health through national public health institutes. Bulletin of the World Health Organization 2005; 83:154-157. 